Spectral Efficiency of Orthogonal Set of Truncated MC CDNA Si gnal s Usi ng Di screte Prol ate Spher oi dal Sequences

\begin{tabular}{|l|l|}
\hline 著者 & Hanamur a Nasanor i , Hyuga Jun \\
\hline j our nal or & W r el ess Commeni cat i ons and Net wor ki ng \\
publ i cat i on t i t l e & Conf er ence, 2008. WCNC 2008. I EEE \\
\hline page range & $980-984$ \\
\hline year & 2008 03-31 \\
\hline URL & ht t p: //hdl . handl e. net /10173/628 \\
\hline
\end{tabular}




\title{
Spectral Efficiency of Orthogonal Set of Truncated MC-CDMA Signals Using Discrete Prolate Spheroidal Sequences
}

\author{
Masanori Hamamura and Jun Hyuga \\ Department of Information Systems Engineering, \\ Kochi University of Technology, Kochi, Japan 782-8502
}

\begin{abstract}
In this paper, we show that a set of multicarrier code-division multiple access (MC-CDMA) signals using a set of discrete prolate spheroidal sequences achieves $95 \%$ of the maximum spectral efficiency at the out-of-band energy of $0.1 \%$ and the bandwidth-duration product of $\mathbf{4 5 . 2 5}$ with an orthogonalizing truncation. It is shown that the density $\rho$ of carriers plays an important role in accurately estimating the out-of-band energy of the signals based on the corresponding eigenvalues and in achieving a small out-of-band energy. We also show that $\rho$ should be chosen such that $\rho \geq 4$ or $\rho \geq 8$. To perform numerically stable eigenanalysis, we employ the technique of singular-value decomposition.
\end{abstract}

\section{INTRODUCTION}

In the design of the spectrally efficient signals, it is necessary to efficiently pack the signals into a given time-frequency plane. The $(\sin x) / x$-shaped Nyquist pulse has a rectangular spectrum that fully occupies a unit frequency slot and provides the maximum spectral efficiency of 2 symbols/s/Hz, the socalled Nyquist efficiency, without any intersymbol interference (ISI) or out-of-band energy. Although it is highly spectrally efficient, the problem for practical use is that such a pulse has eternal, large sidelobes that result in a large amount of energy being spilled out of a given unit time slot. To reduce the duration of the sidelobes without the occurrence of ISI, pulses that have raised-cosine spectra have been commonly considered at the expense of an excess bandwidth [1]. Recently, a "better than" Nyquist pulse that reduces the magnitudes of the largest two sidelobes of pulses having the raised-cosine spectra was proposed [2]. The wavelets have also been considered for use as efficient pulses, since they inherently have the properties of time-frequency localization [3]. For finite-duration pulses, the prolate spheroidal wave function (PSWF) is known to be an optimum pulse that minimizes the out-of-band energy for a given length of time slot [4]-[7].

Another approach for designing efficient finite-duration signals is to employ a set of signals. Orthogonal frequencydivision multiplexing (OFDM) signals [8] are known to be a nearly optimum set of orthogonal signals that can approximately achieve the Nyquist efficiency for a large number of carriers. Each signal in a set of OFDM (pure OFDM) signals has the $(\sin x) / x$-form spectra and hence, has large sidelobes in the frequency domain; however, most of them enter the frequency slots of other carriers in the set, whereby a small total out-of-band energy can be achieved.

Multicarrier code-division multiple access (MC-CDMA) signals can also be a nearly optimum set of orthogonal signals by appropriately choosing code sequences to achieve a small total out-of-band energy. Discrete prolate spheroidal sequences (DPSSs) [7] have been discussed for use with MC-CDMA [9], [10]. In this paper, we discuss the spectral efficiency for a set of finite-duration MC-CDMA signals using the DPSSs and show that an orthogonalizing truncation provides us with a set of signals that achieve higher spectral efficiency than that of the set of OFDM signals for a given identical value of the bandwidth-duration product.

\section{Model of Truncated MC-CDMA Signals}

\section{A. Truncated MC-CDMA Signals}

Let us begin with formulating the shapes of finite-duration MC-CDMA signals using $L$ complex carriers (complex exponentials) with frequencies $\left(l-\frac{1}{2}\right) / T_{0}[\mathrm{~Hz}](l=1,2, \ldots, L)$. The $k$ th signal $c\left(t ; \mathbf{c}_{k}, T\right)(k=1,2, \ldots, K)$ with duration $T[\mathrm{~s}]$ $\left(<T_{0}\right)$ is given by

$$
c\left(t ; \mathbf{c}_{k}, T\right)=g(t ; T) \sum_{l=1}^{L} c_{k, l} e^{j \frac{2 \pi}{T_{0}}\left(l-\frac{1}{2}\right) t},
$$

where $j=\sqrt{-1}, g(t ; T)$ is a rectangular function for the truncation defined as $g(t ; T)=\{1(0<t<T), 0$ (otherwise) $\}$, and $\mathbf{c}_{k}\left(=\left[\begin{array}{lllll}c_{k, 1} & c_{k, 2} & \ldots & c_{k, L}\end{array}\right]^{\mathcal{T}}\right.$; the superscript ${ }^{\mathcal{T}}$ denotes the transpose) is a column vector that contains the code sequence $c_{k, l}(l=1,2, \ldots, L)$ for the $k$ th signal. The signals $c\left(t ; \mathbf{c}_{k}, T\right)$ $(k=1,2, \ldots, K)$ form a set of size $K$.

The $k$ th signal, given by (1), is modulated by the complex message symbol $b_{k}$, which has two message samples in the real and imaginary parts [1] to be transmitted. Let $x_{k}(t ; T)$ be the $k$ th modulated signal $x_{k}(t ; T)=b_{k} c\left(t ; \mathbf{c}_{k}, T\right)$. Therefore, a multiplexed signal $y(t)$ can be represented as

$$
y(t)=\sum_{k=1}^{K} x_{k}(t ; T)=\sum_{k=1}^{K} b_{k} c\left(t ; \mathbf{c}_{k}, T\right) .
$$


For the following discussions, we define a quantity $\rho$, called the density of carriers, as the number of complex carriers within a unit bandwidth $1 / T$, that is,

$$
\rho=T_{0} / T \text {. }
$$

\section{B. Orthogonalization of the Set}

To design an orthogonal set of the signals, we first discuss the cross-correlation $\Phi_{T}\left(\mathbf{c}_{k}, \mathbf{c}_{k^{\prime}}\right)$ between the signals $c\left(t ; \mathbf{c}_{k}, T\right)$ and $c\left(t ; \mathbf{c}_{k^{\prime}}, T\right) . \Phi_{T}\left(\mathbf{c}_{k}, \mathbf{c}_{k^{\prime}}\right)$ is defined as

$$
\Phi_{T}\left(\mathbf{c}_{k}, \mathbf{c}_{k^{\prime}}\right)=\int_{0}^{T} c^{*}\left(t ; \mathbf{c}_{k}, T\right) c\left(t ; \mathbf{c}_{k^{\prime}}, T\right) d t,
$$

where the superscript $*$ denotes the complex conjugate.

Substituting (1) into (4) yields

$$
\begin{aligned}
& \Phi_{T}\left(\mathbf{c}_{k}, \mathbf{c}_{k^{\prime}}\right) \\
& =\int_{0}^{T}\left(\sum_{l=1}^{L} c_{k, l}^{*} e^{-j \frac{2 \pi}{T_{0}}\left(l-\frac{1}{2}\right) t}\right)\left(\sum_{m=1}^{L} c_{k^{\prime}, m} e^{j \frac{2 \pi}{T_{0}}\left(m-\frac{1}{2}\right) t}\right) d t \\
& =T_{0} \sum_{l=1}^{L} \sum_{m=1}^{L} c_{k, l}^{*} r_{l, m} c_{k^{\prime}, m},
\end{aligned}
$$

where $r_{l, m}$ is the cross-correlation between the complex carriers $f_{l}(t ; T)=g(t ; T) \frac{1}{\sqrt{T_{0}}} \exp j \frac{2 \pi(l-1 / 2)}{T_{0}} t$ and $f_{m}(t ; T)=$ $g(t ; T) \frac{1}{\sqrt{T_{0}}} \exp j \frac{2 \pi(m-1 / 2)}{T_{0}} t$, which is given by

$$
\begin{aligned}
& r_{l, m}=\int_{0}^{T} f_{l}^{*}(t ; T) f_{m}(t ; T) d t \\
& =\left\{\begin{array}{ll}
1 / \rho & (l=m) \\
\frac{1}{\rho} \frac{\sin ((l-m) \pi / \rho)}{(l-m) \pi / \rho} e^{-j(l-m) \pi / \rho} & (l \neq m)
\end{array} .\right.
\end{aligned}
$$

Let $\mathbf{R}_{\rho}$ be an $L$-by- $L$ matrix, whose $(l, m)$ th entry is $r_{l, m}$, such that

$$
\mathbf{R}_{\rho}=\left[r_{l, m}\right] .
$$

Since it is obvious from (6) that $r_{l, m}^{*}=r_{m, l}$, the matrix $\mathbf{R}_{\rho}$ is Hermitian; thus, the relation $\mathbf{R}_{\rho}^{\mathcal{H}}=\mathbf{R}_{\rho}$ holds, where the superscript ${ }^{\mathcal{H}}$ denotes the Hermitian transpose (the transpose combined with the complex conjugate).

By using the matrix $\mathbf{R}_{\rho}$ and the code sequence $\mathbf{c}_{k}$, the crosscorrelation (5) can be rewritten as

$$
\Phi_{T}\left(\mathbf{c}_{k}, \mathbf{c}_{k^{\prime}}\right)=T_{0} \mathbf{c}_{k}^{\mathcal{H}} \mathbf{R}_{\rho} \mathbf{c}_{k^{\prime}}
$$

The matrix $\mathbf{R}_{\rho}$ in (8) can be decomposed into its eigenvalues $\lambda_{\rho, i}$ and corresponding eigenvectors $\mathbf{v}_{\rho, i}$, which satisfy $\mathbf{R}_{\rho} \mathbf{v}_{\rho, i}=\lambda_{\rho, i} \mathbf{v}_{\rho, i}\left(i=1,2, \ldots, K^{\prime} ; K^{\prime}\right.$ is the rank of the matrix $\mathbf{R}_{\rho}$, and $\left.K^{\prime} \leq L\right)$, such that

$$
\mathbf{R}_{\rho}=\sum_{i=1}^{K^{\prime}} \lambda_{\rho, i} \mathbf{v}_{\rho, i} \mathbf{v}_{\rho, i}^{\mathcal{H}}
$$

where the eigenvalues $\lambda_{\rho, i}$ have values $0 \leq \lambda_{\rho, i} \leq 1$, and the eigenvectors $\mathbf{v}_{\rho, i}\left(=\left[\begin{array}{lllll}v_{\rho, i, 1} & v_{\rho, i, 2} & \ldots & v_{\rho, i, L}\end{array}\right]^{\mathcal{T}}\right)$ have a unit norm (i.e., $\left\|\mathbf{v}_{\rho, i}\right\|=\left(\mathbf{v}_{\rho, i}^{\mathcal{H}} \mathbf{v}_{\rho, i}\right)^{\frac{1}{2}}=1$ ) and are orthogonal to each other (i.e., $\left.\mathbf{v}_{\rho, i}^{\mathcal{H}} \mathbf{v}_{\rho, i^{\prime}}=\left\{1\left(i=i^{\prime}\right), 0\left(i \neq i^{\prime}\right)\right\}\right)$. To obtain numerically stable results for the eigenanalysis of the matrix $\mathbf{R}_{\rho}$, we employ the technique of singular-value decomposition (see Appendix I). Without loss of generality, we assume that the eigenvalues are numbered such that $\lambda_{\rho, 1} \geq \lambda_{\rho, 2} \geq \ldots \geq$ $\lambda_{\rho, K^{\prime}}$.

Substituting (9) into (8), we have

$$
\Phi_{T}\left(\mathbf{c}_{k}, \mathbf{c}_{k^{\prime}}\right)=T_{0} \mathbf{c}_{k}^{\mathcal{H}}\left(\sum_{i=1}^{K^{\prime}} \lambda_{\rho, i} \mathbf{v}_{\rho, i} \mathbf{v}_{\rho, i}^{\mathcal{H}}\right) \mathbf{c}_{k^{\prime}} .
$$

Therefore, by choosing the vector $\mathbf{c}_{\rho, k}$, which is given by

$$
\mathbf{c}_{\rho, k}=\frac{1}{\sqrt{\lambda_{\rho, k}}} \mathbf{v}_{\rho, k} \Longrightarrow \mathbf{c}_{k},
$$

as the code sequence vector $\mathbf{c}_{k}$ for the $k$ th signal $(k=$ $1,2, \ldots, K ; K \leq K^{\prime}$ ), and by substituting (11) into (10), we have

$$
\begin{aligned}
& \Phi_{T}\left(\mathbf{c}_{\rho, k}, \mathbf{c}_{\rho, k^{\prime}}\right) \\
& =T_{0} \frac{1}{\sqrt{\lambda_{\rho, k}}} \mathbf{v}_{\rho, k}^{\mathcal{H}}\left(\sum_{i=1}^{K^{\prime}} \lambda_{\rho, i} \mathbf{v}_{\rho, i} \mathbf{v}_{\rho, i}^{\mathcal{H}}\right) \frac{1}{\sqrt{\lambda_{\rho, k^{\prime}}}} \mathbf{v}_{\rho, k^{\prime}} \\
& =\left\{\begin{array}{ll}
T_{0} & \left(k=k^{\prime}\right) \\
0 & \left(k \neq k^{\prime}\right)
\end{array} .\right.
\end{aligned}
$$

Expression (12) indicates that the signals $c\left(t ; \mathbf{c}_{\rho, k}, T\right)(k=$ $\left.1,2, \ldots, K ; K \leq K^{\prime}\right)$ are orthogonal for the duration $0<t<$ $T$. We employ the set of vectors $\mathbf{c}_{\rho, k}$ as the code sequences [10], which is a complex version of the set of DPSSs, ${ }^{1}$ and discuss the signals transmitted in the duration $0<t<T$.

\section{Symbol Detection}

The message symbol $b_{k}$ that modulates the $k$ th signal $c\left(t ; \mathbf{c}_{\rho, k}, T\right)$ can easily be detected from the multiplexed signal $y(t)$ using the corresponding correlator (or equivalently, using the matched filter that has the impulse response $h_{k}(\tau)=$ $\left.c^{*}\left(T-\tau ; \mathbf{c}_{\rho, k}, T\right)\right)$.

The $k$ th correlator output $z_{k}(T)$ at time $t=T$ is given by

$$
z_{k}(T)=\int_{0}^{T} c^{*}\left(t ; \mathbf{c}_{\rho, k}, T\right) y(t) d t .
$$

Substituting (2) into (13), and using (12), we obtain

$$
\begin{aligned}
z_{k}(T) & =\int_{0}^{T} c^{*}\left(t ; \mathbf{c}_{\rho, k}, T\right)\left(\sum_{k^{\prime}=1}^{K} b_{k^{\prime}} c\left(t ; \mathbf{c}_{\rho, k^{\prime}}, T\right)\right) d t \\
& =\sum_{k^{\prime}=1}^{K} b_{k^{\prime}} \Phi_{T}\left(\mathbf{c}_{\rho, k}, \mathbf{c}_{\rho, k^{\prime}}\right)=b_{k} T_{0}
\end{aligned}
$$

${ }^{1}$ For an $L$-by- $L$ diagonal matrix $\mathbf{D}=\operatorname{diag}\left\{e^{j\left(1-\frac{1}{2}\right) \pi / \rho}, e^{j\left(2-\frac{1}{2}\right) \pi / \rho}\right.$, $\left.\ldots, e^{j\left(L-\frac{1}{2}\right) \pi / \rho}\right\}\left(\mathbf{D}^{-1}=\mathbf{D}^{\mathcal{H}}\right)$, the set of DPSSs is typically defined as the real solution of the set of vectors $\mathbf{d}_{\rho, k}$ that satisfy $\left(\mathbf{D} \mathbf{R}_{\rho} \mathbf{D}^{\mathcal{H}}\right) \mathbf{d}_{\rho, k}=$ $\lambda_{\rho, k} \mathbf{d}_{\rho, k}$ for distinct eigenvalues $\lambda_{\rho, k}$ [7]. It is easily verified that $\mathbf{d}_{\rho, k}$ can be expressed as the form $\mathbf{d}_{\rho, k}=\mathbf{D} \mathbf{v}_{\rho, k}$; thus, $\mathbf{v}_{\rho, k}=\mathbf{D}^{\mathcal{H}} \mathbf{d}_{\rho, k}$. To produce the orthogonal set of signals defined in the duration $-T / 2<t<$ $T / 2$, the vectors $\mathbf{d}_{\rho, k}$ can be employed as the code sequences regardless of the real or complex solutions. 
Note that the correlator (or matched filter) provides us with an optimum method of detection that achieves the highest signal-to-noise ratio (SNR) over additive white Gaussian noise (AWGN) channels.

\section{SPECTRAL EFFICIENCY}

\section{A. Out-of-Band Energy}

A reliable value of out-of-band energy is required to accurately evaluate the spectral efficiency of the set of truncated MC-CDMA signals. Since the out-of-band energy $E_{k}^{(\text {out })}$ of the signal $x_{k}(t ; T)$ can be obtained from the total energy $E_{k}^{\text {(total) }}$ minus the in-band energy $E_{k}^{(\text {in })}$, we first discuss the in-band energy $E_{k}^{(\mathrm{in})}$.

Here, we show that approximately $100 \lambda_{k}[\%]$ of the energy of $x_{k}(t ; T)$ occupies the bandwidth $W=L / T_{0}$, defined in the frequency band $B=\left[0, L / T_{0}\right]$.

Let $X_{k}(f ; T)\left(=\mathscr{F}\left[x_{k}(t ; T)\right]\right)$ be the Fourier transform of $x_{k}(t ; T) . X_{k}(f ; T)$ is given by

$$
\begin{aligned}
X_{k}(f ; T) & =\int_{-\infty}^{\infty} x_{k}(t ; T) e^{-j 2 \pi f t} d t \\
& =b_{k} \int_{0}^{T} c\left(t ; \mathbf{c}_{\rho, k}, T\right) e^{-j 2 \pi f t} d t \\
& =b_{k} T_{0} \sum_{l=1}^{L} c_{\rho, k, l} r_{l}^{*}(f),
\end{aligned}
$$

where $r_{l}^{*}(f)$ denotes

$$
\begin{aligned}
r_{l}^{*}(f) & =\frac{1}{T_{0}} \int_{0}^{T} e^{-j 2 \pi f t} e^{j \frac{2 \pi}{T_{0}}\left(l-\frac{1}{2}\right) t} d t \\
& =\frac{1}{\rho} \frac{\sin \left(\left(T_{0} f-l+\frac{1}{2}\right) \pi / \rho\right)}{\left(T_{0} f-l+\frac{1}{2}\right) \pi / \rho} e^{-j\left(T_{0} f-l+\frac{1}{2}\right) \pi / \rho} .
\end{aligned}
$$

The in-band energy $E_{k}^{(\mathrm{in})}$ of $x_{k}(t ; T)$ can be evaluated using (15) as follows:

$$
\begin{aligned}
E_{k}^{(\mathrm{in})} & =\int_{B}\left|X_{k}(f ; T)\right|^{2} d f \\
& =\left|b_{k}\right|^{2} T_{0}^{2} \int_{0}^{L / T_{0}}\left|\sum_{l=1}^{L} c_{\rho, k, l} r_{l}^{*}(f)\right|^{2} d f .
\end{aligned}
$$

Now, we approximate the integral in (17) by a discrete summation of the areas of $L$ rectangles with the frequency interval $\Delta_{f}=1 / T_{0}$, that is,

$$
\tilde{E}_{k}^{(\text {in })}=\left.\left|b_{k}\right|^{2} T_{0}^{2} \sum_{m=1}^{L}\left|\sum_{l=1}^{L} c_{\rho, k, l} r_{l}^{*}(f)\right|_{f=\frac{m-1 / 2}{T_{0}}}\right|^{2} \Delta_{f} .
$$

Expression (18) can be a good approximation for obtaining the exact values of $E_{k}^{(\mathrm{in})}$ when $\Delta_{f}\left(=1 / T_{0}\right) \rightarrow 0$, or $\rho(=$ $\left.T_{0} / T\right) \rightarrow \infty$ for a certain fixed value of the duration $T$ of the signals, and the approximation is independent of the length $L$ of the code sequence. It will be shown that (18) can be a good approximation only when $\rho \geq 4$ or $\rho \geq 8$.

We now determine the approximated quantity $\tilde{E}_{k}^{(\text {in) }}$ of the in-band energy.
Note that $r_{l}^{*}(f)$, given by (16), coincides with the complex conjugate $r_{l, m}^{*}$ of $r_{l, m}$, given by (6), at the frequencies $f=$ $\left(m-\frac{1}{2}\right) / T_{0}(m=1,2, \ldots, L)$.

$$
\left.r_{l}^{*}(f)\right|_{f=\frac{m-1 / 2}{T_{0}}}=r_{l, m}^{*}
$$

Therefore, the following relation holds:

$$
\begin{aligned}
\left.\sum_{l=1}^{L} c_{\rho, k, l} r_{l}^{*}(f)\right|_{f=\frac{m-1 / 2}{T_{0}}} & =\sum_{l=1}^{L} c_{\rho, k, l} r_{l, m}^{*} \\
& =\mathbf{r}_{m}^{\mathcal{H}} \mathbf{c}_{\rho, k},
\end{aligned}
$$

where $\mathbf{r}_{m}$ denotes the $m$ th column vector of the Hermitian matrix $\mathbf{R}_{\rho}\left(=\left[\mathbf{r}_{1} \mathbf{r}_{2} \ldots \mathbf{r}_{m} \ldots \mathbf{r}_{L}\right] ; \mathbf{R}_{\rho}^{\mathcal{H}}=\mathbf{R}_{\rho}\right)$.

Substituting (20) into (18) yields

$$
\begin{aligned}
\tilde{E}_{k}^{(\mathrm{in})} & =\left|b_{k}\right|^{2} T_{0}^{2} \sum_{m=1}^{L}\left|\mathbf{r}_{m}^{\mathcal{H}} \mathbf{c}_{\rho, k}\right|^{2} \Delta_{f} \\
& =\left|b_{k}\right|^{2} T_{0}^{2}\left\|\mathbf{R}_{\rho}^{\mathcal{H}} \mathbf{c}_{\rho, k}\right\|^{2} \Delta_{f},
\end{aligned}
$$

where $\|\cdot\|$ denotes the norm of the enclosed vector.

Finally, upon substituting (9) for $\mathbf{R}_{\rho}$ and (11) for $\mathbf{c}_{\rho, k}$ into expression (21), we arrive at the desired approximation $\tilde{E}_{k}^{(\text {in })}$ for the in-band energy of the signal $x_{k}(t ; T)$.

$$
\begin{aligned}
\tilde{E}_{k}^{(\mathrm{in})} & =\left|b_{k}\right|^{2} T_{0}^{2}\left\|\left(\sum_{i=1}^{K^{\prime}} \lambda_{\rho, i} \mathbf{v}_{\rho, i} \mathbf{v}_{\rho, i}^{\mathcal{H}}\right) \mathbf{c}_{\rho, k}\right\|^{2} \Delta_{f} \\
& =\left|b_{k}\right|^{2} T_{0}^{2}\left\|\sqrt{\lambda_{\rho, k}} \mathbf{v}_{\rho, k}\right\|^{2} \Delta_{f} \\
& =\left|b_{k}\right|^{2} T_{0} \lambda_{\rho, k}
\end{aligned}
$$

According to Parseval's theorem, the total energy $E_{k}^{(\text {total })}$ of $X_{k}(f ; T)$ in the frequency domain is identical to that of $x_{k}(t ; T)$ in the time domain, that is,

$$
\begin{aligned}
E_{k}^{(\text {total })} & =\int_{-\infty}^{\infty}\left|X_{k}(f ; T)\right|^{2} d f \\
& =\int_{0}^{T}\left|x_{k}(t ; T)\right|^{2} d t \\
& =\left|b_{k}\right|^{2} \int_{0}^{T} c^{*}\left(t ; \mathbf{c}_{\rho, k}, T\right) c\left(t ; \mathbf{c}_{\rho, k}, T\right) d t \\
& =\left|b_{k}\right|^{2} \Phi_{T}\left(\mathbf{c}_{\rho, k}, \mathbf{c}_{\rho, k}\right) .
\end{aligned}
$$

Using (12), we obtain

$$
E_{k}^{\text {(total) }}=\left|b_{k}\right|^{2} T_{0} .
$$

The out-of-band energy $E_{k}^{\text {(out) }}$ is obtained using (22) and (24) as

$$
\begin{aligned}
E_{k}^{(\text {out })} & =E_{k}^{(\text {total })}-E_{k}^{(\text {in })} \\
& \approx E_{k}^{\text {(total) }}-\tilde{E}_{k}^{(\text {in })}=\left|b_{k}\right|^{2} T_{0}\left(1-\lambda_{\rho, k}\right) .
\end{aligned}
$$

Thus, it is seen from (24) to (26) that approximately 100(1$\left.\lambda_{\rho, k}\right)[\%]$ of the energy of the signal $x_{k}(t ; T)$ exists outside the given frequency band $B$.

Figure 1 shows the relationship between the numerically evaluated exact values of the out-of-band energies and those 


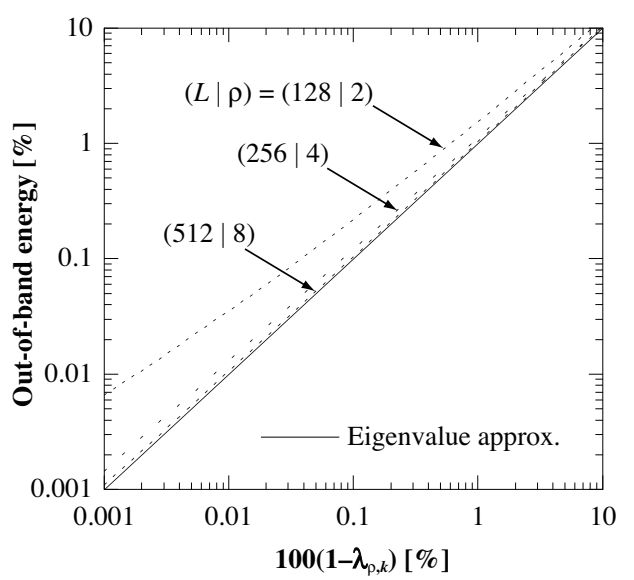

Fig. 1. Relationship between exact value of the out-of-band energy of $x_{k}(t ; T)$ and eigenvalue approximation based on $(26)(L / \rho=64(=W T))$.

obtained using approximation (26). The solid line shows the approximation based on (26), and dotted lines show the values obtained from (17), (24), and (25).

As can be observed in Fig. 1, the approximation is valid only for $\rho \geq 4$ or more precisely, for $\rho \geq 8$. In addition, it is important to note that we must choose a large value of density $\rho$ such that $\rho \geq 4$ or $\rho \geq 8$ to produce the set of signals $x_{k}(t ; T)$ that achieve a smaller out-of-band energy.

\section{B. Spectral Efficiency}

Since each symbol $b_{k}$ takes a complex value containing two message samples in its real and imaginary parts, the multiplexed signal $y(t)\left(=\sum_{k=1}^{K} x_{k}(t ; T)=\sum_{k=1}^{K} b_{k} c\left(t ; \mathbf{c}_{\rho, k}, T\right)\right)$ of size $K$ conveys $2 K$ samples during $T$ [s] using the bandwidth $W=L / T_{0}=L /(\rho T)[\mathrm{Hz}]$. Therefore, the spectral efficiency $\eta$ [samples/s/Hz] of $y(t)$ is given by

$$
\eta=\frac{2 K \frac{1}{T}}{W}=\frac{2 K}{W T}\left(=\frac{2 K}{L / \rho}\right) .
$$

To determine the size $K$ of the set of the signals, we consider the out-of-band energy for the multiplexed signal $y(t)$. For simplicity, we assume that $\mathrm{E}\left[b_{k}^{*} b_{k^{\prime}}\right]=\{\mathcal{E}(k=$ $\left.\left.k^{\prime}\right), 0\left(k \neq k^{\prime}\right)\right\}$ (E[.] denotes the ensemble average). For this case, the average out-of-band energy $\mathscr{E}_{y}^{\text {(out) }}$ for the multiplexed signal $y(t)$ can be evaluated using the approximation in (26) as

$$
\begin{aligned}
\mathscr{E}_{y}^{(\text {out })} & =\mathrm{E}\left[\sum_{k=1}^{K} E_{k}^{(\text {out })}\right]=\sum_{k=1}^{K} \mathrm{E}\left[E_{k}^{(\text {total })}-E_{k}^{(\text {in })}\right] \\
& \approx \sum_{k=1}^{K} \mathrm{E}\left[\left|b_{k}\right|^{2}\right] T_{0}\left(1-\lambda_{\rho, k}\right) \\
& =K \mathcal{E} T_{0}\left(1-\bar{\lambda}_{\rho}\right),
\end{aligned}
$$

where $\bar{\lambda}_{\rho}$ is the average of the eigenvalues $\lambda_{\rho, k}$, defined as

$$
\bar{\lambda}_{\rho}=\frac{1}{K} \sum_{k=1}^{K} \lambda_{\rho, k} .
$$

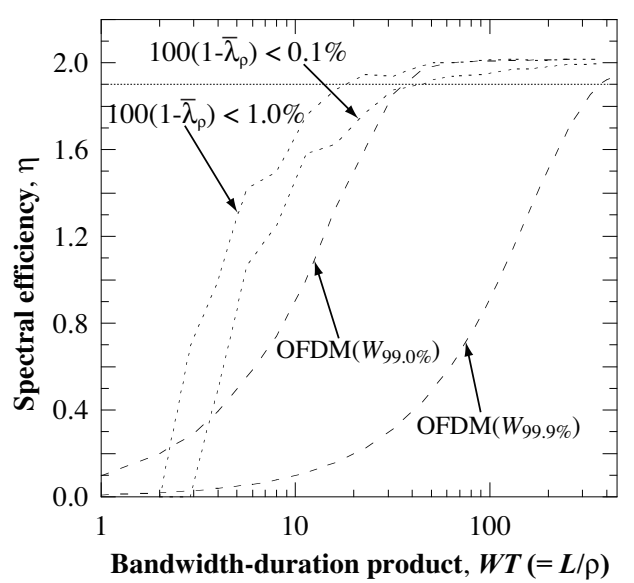

Fig. 2. Spectral efficiency $\eta$ vs bandwidth-duration product $W T(\rho=8)$.

On the other hand, using (24), the total energy $\mathscr{E}_{y}^{(\text {total })}$ of $y(t)$ is

$$
\begin{aligned}
\mathscr{E}_{y}^{(\text {total })}=\mathrm{E}\left[\sum_{k=1}^{K} E_{k}^{(\text {total })}\right] & =\sum_{k=1}^{K} \mathrm{E}\left[\left|b_{k}\right|^{2}\right] T_{0} \\
& =K \mathcal{E} T_{0} .
\end{aligned}
$$

Thus, from (28) and (30), the out-of-band energy of $y(t)$ is $100 \mathscr{E}_{y}^{\text {(out) }} / \mathscr{E}_{y}^{\text {(total) }} \approx 100\left(1-\bar{\lambda}_{\rho}\right)[\%]$. Note that we can obtain reliable values of the average out-of-band energy $\mathscr{E}_{y}^{\text {(out) }}$ by calculating $\bar{\lambda}_{\rho}$, given by (29), only when we choose a large value of density $\rho$ for the set of signals.

Let $K_{100 p}$ be the maximum size of $K$ for which $y(t)$ satisfies $100\left(1-\bar{\lambda}_{\rho}\right)<100 p[\%]$, that is,

$$
1-\frac{1}{K_{100 p}} \sum_{k=1}^{K_{100 p}} \lambda_{\rho, k}<p \leq 1-\frac{1}{K_{100 p}+1} \sum_{k=1}^{K_{100 p}+1} \lambda_{\rho, k} .
$$

It should be pointed out that $K_{100 p}$ depends not only on the value of $100 p$ but also on the values of $L$ and $\rho$. We employ $K_{100 p}$ as the size $K$ in (27) for the evaluation of the spectral efficiency of $y(t)$. Results are shown in Fig. 2 in comparison with those of OFDM (pure OFDM) signals, for which $99.0 \%$ bandwidth $\left(W_{99.0 \%}\right)$ and $99.9 \%$ bandwidth $\left(W_{99.9 \%}\right)$ are considered.

As can be observed in Fig. 2, the spectral efficiency increases with the bandwidth-duration product $W T$. For the case of $100\left(1-\bar{\lambda}_{\rho}\right)<0.1 \%$, the multiplexed signal $y(t)$ achieves $\eta \approx 1.90$, which corresponds to $95 \%$ of the maximum spectral efficiency of the Nyquist pulse $(\sin t) / t$, at $W T=45.25{ }^{2}$ whereas the set of OFDM signals requires $W T=373$ for the out-of-band energy of $0.1 \%$ (the curve for $W_{99.9 \%}$ ) to achieve $\eta \approx 1.90$.

The energy density spectra of $y(t)$ for different sizes of $K$ are shown in Fig. 3 for $(L \mid \rho)=(362 \mid 8)$. It is observed that

\footnotetext{
${ }^{2} K_{0.1 \%}=43$ was obtained at $(L \mid \rho)=(362 \mid 8)$, which corresponds to $W T=45.25$. For this case, the approximate value of the average out-ofband energy was $100\left(1-\bar{\lambda}_{\rho}\right)=0.057 \%$, while the numerically evaluated exact value was $0.059 \%$. Both of these values are less than the prescribed value of $100 p=0.1 \%$.
} 


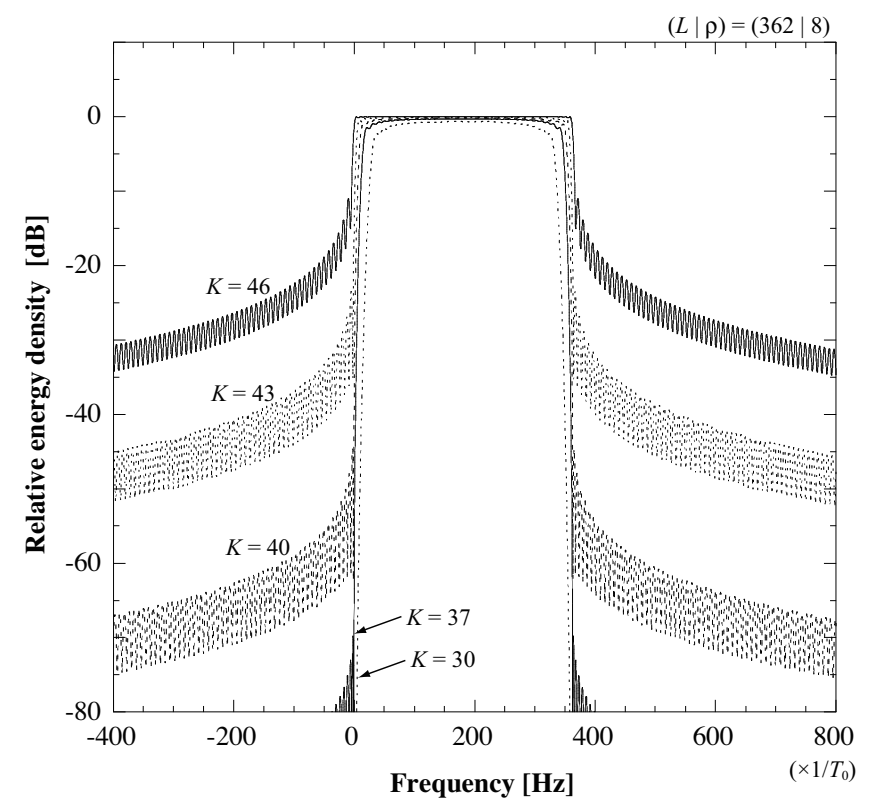

Fig. 3. Energy density spectra of $y(t)$ for different sizes of $K$ for $(L \mid \rho)=$ $(362 \mid 8)$ (i.e., $W T=45.25)$.

the out-of-band characteristic of $y(t)$ can be controlled by the size $K$.

\section{CONCLUSION}

The spectral efficiency of an orthogonal set of truncated MC-CDMA signals using DPSSs has been discussed. It has been shown that the set of signals can be more efficiently packed into a limited space of the time-frequency plane than the set of OFDM signals.

\section{APPENDIX I \\ EIGENANALYSIS OF MATRIX $\mathbf{R}_{\rho}$}

The purpose of our eigenanalysis is to obtain the eigenvalues $\lambda_{\rho, i}$ and corresponding eigenvectors $\mathbf{v}_{\rho, i}\left(i=1,2, \ldots, K^{\prime}\right)$ such that expression (9) holds.

Let $\mathbf{f}_{\rho, l}$ and $\mathbf{f}_{\rho, m}$ be column vectors

$$
\begin{aligned}
\mathbf{f}_{\rho, l} & =\left[\begin{array}{llll}
f_{\rho, l, 1} & f_{\rho, l, 2} & \ldots & f_{\rho, l, M_{T}}
\end{array}\right]^{\mathcal{T}} \\
\mathbf{f}_{\rho, m} & =\left[\begin{array}{llll}
f_{\rho, m, 1} & f_{\rho, m, 2} & \ldots & f_{\rho, m, M_{T}}
\end{array}\right]^{\mathcal{T}}
\end{aligned}
$$

that consist of discrete-time samples $f_{\rho, l, n}$ and $f_{\rho, m, n}(n=$ $\left.1,2, \ldots, M_{T}\right)$ of the complex carriers $f_{l}(t ; T)$ and $f_{m}(t ; T)$, defined as ${ }^{3}$

$$
\begin{aligned}
f_{\rho, l, n} & =\frac{1}{\sqrt{\rho M_{T}}} \exp j \frac{2 \pi(l-1 / 2)(n-1)}{\rho M_{T}}, \\
f_{\rho, m, n} & =\frac{1}{\sqrt{\rho M_{T}}} \exp j \frac{2 \pi(m-1 / 2)(n-1)}{\rho M_{T}} .
\end{aligned}
$$

The $(l, m)$ th entry $r_{l, m}$, given by (6), of the matrix $\mathbf{R}_{\rho}$ can be represented using $\mathbf{f}_{\rho, l}$ and $\mathbf{f}_{\rho, m}$ as $r_{l, m} \approx \mathbf{f}_{\rho, l}^{\mathcal{H}} \mathbf{f}_{\rho, m}\left(\triangleq \hat{r}_{l, m}\right)$.

Let us define a new matrix $\hat{\mathbf{F}}_{\rho}=\left[\begin{array}{llll}\mathbf{f}_{\rho, 1} & \mathbf{f}_{\rho, 2} & \ldots & \mathbf{f}_{\rho, L}\end{array}\right] . \mathbf{R}_{\rho}$ can be represented using $\hat{\mathbf{F}}_{\rho}$ as $\mathbf{R}_{\rho} \approx \hat{\mathbf{F}}_{\rho}^{\mathcal{H}} \hat{\mathbf{F}}_{\rho}\left(\triangleq \hat{\mathbf{R}}_{\rho}\right)$.

\footnotetext{
${ }^{3}$ We employ $M_{T}=4 L$ for the numerical discussion throughout the paper.
}

In accordance with the technique of singular-value decomposition [11], $\hat{\mathbf{F}}_{\rho}$ is decomposed into

$$
\hat{\mathbf{F}}_{\rho}=\hat{\mathbf{U}}_{\rho} \hat{\boldsymbol{\Lambda}}_{\rho}^{\frac{1}{2}} \hat{\mathbf{V}}_{\rho}^{\mathcal{H}},
$$

where $\hat{\boldsymbol{\Lambda}}_{\rho}^{\frac{1}{2}}=\operatorname{diag}\left\{\hat{\lambda}_{\rho, 1}^{\frac{1}{2}}, \hat{\lambda}_{\rho, 2}^{\frac{1}{2}}, \ldots, \hat{\lambda}_{\rho, \hat{K}^{\prime}}^{\frac{1}{2}}\right\}$ is a $\hat{K}^{\prime}$-by- $\hat{K}^{\prime}$ diagonal matrix that contains $\hat{K}^{\prime}$ distinct singular values $\hat{\lambda}_{\rho, i}^{\frac{1}{2}}$ $\left(i=1,2, \ldots, \hat{K}^{\prime} ; \hat{K}^{\prime}\right.$ is the rank of $\hat{\mathbf{F}}_{\rho}$ and $\operatorname{rank}\left(\hat{\mathbf{F}}_{\rho}\right) \stackrel{\lambda^{2}}{=}$ $\left.\operatorname{rank}\left(\hat{\mathbf{R}}_{\rho}\right) \leq \min \left(L, M_{T}\right)\right), \hat{\mathbf{U}}_{\rho}=\left[\begin{array}{llll}\hat{\mathbf{u}}_{\rho, 1} & \hat{\mathbf{u}}_{\rho, 2} & \ldots & \hat{\mathbf{u}}_{\rho, \hat{K}^{\prime}}\end{array}\right]$ and $\hat{\mathbf{V}}_{\rho}=\left[\begin{array}{llll}\hat{\mathbf{v}}_{\rho, 1} & \hat{\mathbf{v}}_{\rho, 2} & \ldots & \hat{\mathbf{v}}_{\rho, \hat{K}^{\prime}}\end{array}\right]$ are an $M_{T}$-by- $\hat{K}^{\prime}$ and an $L$-by$\hat{K}^{\prime}$ matrix, respectively, where $\hat{\mathbf{u}}_{\rho, i}^{\mathcal{H}} \hat{\mathbf{u}}_{\rho, i^{\prime}}=\left\{1\left(i=i^{\prime}\right), 0(i \neq\right.$ $\left.\left.i^{\prime}\right)\right\}$ and $\hat{\mathbf{v}}_{\rho, i}^{\mathcal{H}} \hat{\mathbf{v}}_{\rho, i^{\prime}}=\left\{1\left(i=i^{\prime}\right), 0\left(i \neq i^{\prime}\right)\right\}$ hold.

Using (32) and the relations $\hat{\mathbf{U}}_{\rho}^{\mathcal{H}} \hat{\mathbf{U}}_{\rho}=\mathbf{1}_{\hat{K}^{\prime} \times \hat{K}^{\prime}}\left(\hat{K}^{\prime}\right.$-by- $\hat{K}^{\prime}$ identity matrix) and $\hat{\boldsymbol{\Lambda}}_{\rho}^{\frac{1}{2}} \hat{\boldsymbol{\Lambda}}_{\rho}^{\frac{1}{2}}=\hat{\boldsymbol{\Lambda}}_{\rho}, \hat{\mathbf{R}}_{\rho}$ can be expressed as

$$
\begin{aligned}
\hat{\mathbf{R}}_{\rho}\left(=\hat{\mathbf{F}}_{\rho}^{\mathcal{H}} \hat{\mathbf{F}}_{\rho}\right) & =\left(\hat{\mathbf{U}}_{\rho} \hat{\boldsymbol{\Lambda}}_{\rho}^{\frac{1}{2}} \hat{\mathbf{V}}_{\rho}^{\mathcal{H}}\right)^{\mathcal{H}}\left(\hat{\mathbf{U}}_{\rho} \hat{\boldsymbol{\Lambda}}_{\rho}^{\frac{1}{2}} \hat{\mathbf{V}}_{\rho}^{\mathcal{H}}\right) \\
& =\hat{\mathbf{V}}_{\rho} \hat{\boldsymbol{\Lambda}}_{\rho}^{\frac{1}{2}} \hat{\mathbf{U}}_{\rho}^{\mathcal{H}} \hat{\mathbf{U}}_{\rho} \hat{\boldsymbol{\Lambda}}_{\rho}^{\frac{1}{2}} \hat{\mathbf{V}}_{\rho}^{\mathcal{H}}=\hat{\mathbf{V}}_{\rho} \hat{\boldsymbol{\Lambda}}_{\rho} \hat{\mathbf{V}}_{\rho}^{\mathcal{H}} \\
& =\sum_{i=1}^{\hat{K}^{\prime}} \hat{\lambda}_{\rho, i} \hat{\mathbf{v}}_{\rho, i} \hat{\mathbf{v}}_{\rho, i}^{\mathcal{H}} .
\end{aligned}
$$

Expression (33) corresponds to the result of the objective eigenanalysis (9). Thus, the eigenvalues $\hat{\lambda}_{\rho, i}$ and corresponding eigenvectors $\hat{\mathbf{v}}_{\rho, i}$ of $\hat{\mathbf{R}}_{\rho}$ can be obtained by the singularvalue decomposition of matrix $\hat{\mathbf{F}}_{\rho}$.

\section{ACKNOWLEDGMENT}

This work was supported in part by a Grant-in-Aid for Young Scientists (B19760271) from the Ministry of Education, Culture, Sports, Science and Technology (MEXT) in Japan.

\section{REFERENCES}

[1] J. G. Proakis, Digital Communications (3rd ed.), McGraw-Hill, New York, 1995.

[2] N. C. Beaulieu,, C. C. Tan, and M. O. Damen, "A "better than" Nyquist pulse," IEEE Commun. Lett., vol.5, no.9, pp.367-368, Sept. 2001.

[3] P. P. Gandhi, S. S. Rao, and R. S. Pappu, "Wavelets for waveform coding of digital symbols," IEEE Trans. Signal Processing, vol.45, no.9, pp.2387-2390, Sept. 1997.

[4] J. H. H. Chalk, "The optimum pulse-shape for pulse communication," Proc. IEE, Radio and Communication Engineering, vol.87, pp.88-92, 1950.

[5] D. Slepian and H. O. Pollak, "Prolate spheroidal wave functions, Fourier analysis and uncertainty - I," Bell Syst. Tech. J., vol.40, pp.43-63, Jan. 1961.

[6] H. J. Landau and H. O. Pollak, "Prolate spheroidal wave functions, Fourier analysis and uncertainty - II," Bell Syst. Tech. J., vol.40, pp.6584, Jan. 1961.

[7] D. Slepian, "Prolate spheroidal wave functions, Fourier analysis and uncertainty - V: The discrete case," Bell Syst. Tech. J., vol.57, no.5, pp.1371-1430, May-June 1978.

[8] R. van Nee and R. Prasad, OFDM for Wireless Multimedia Communications, Artech House, 2000.

[9] I. Raos, S. Zazo, and J. M. Paez-Berrallo, "Reduced interference MCCDMA system using discrete prolate codes," IEEE ICASSP '02, vol.3, pp.2597-2600, May 2002.

[10] I. Raos, S. Zazo, and F. Bader, "Prolate spheroidal functions: A general framework for MC-CDMA waveforms without time redundancy," IEEE PIMRC 2002, vol.5, pp.2342-2346, Sept. 2002.

[11] S. Haykin, Adaptive Filter Theory (3rd ed.), Prentice Hall, 1996. 